\title{
New Ideology of All-Optical Microwave Systems Based on the Use of Semiconductor Laser as a Down-Converter.
}

\author{
V. B. GORFINKEL, ${ }^{*}$ M.I. GOUZMAN ${ }^{* *}$, S. LURYI ${ }^{*}$ and E.L. PORTNOI ${ }^{* * *}$ \\ ${ }^{*}$ State University of New York, Stony Brook, NY 11794-2350 \\ ${ }^{* * *}$ Bally Wulff GmbH, D-34125, Kassel, Germany \\ ${ }^{* * * *}$ Ioffe Institute, 194026, St. Petersburg, Russia
}

\begin{abstract}
We propose a novel all-optical structure of phase lock loop for locking two semiconductor lasers with a stable microwave offset for use in phased-array antenna systems.
\end{abstract}

\section{Motivation}

Optical control of millimeter wave antenna arrays is an important technological challenge. This goal requires several key elements, currently under intense development worldwide. One of these elements is a dual optical beam source capable of producing a stable millimeter wave beat frequency when mixed at an antenna site. Such a source is important in the implementation of virtually any architecture for optical distribution of microwave phase between antenna array oscillators.

Development of a universal source based on semiconductor lasers faces several essential barriers. Semiconductor lasers have a relatively broad linewidth, and moreover the line center slowly drifts around due to environmental influences. This places a particularly stringent demand on the stabilizing phase lock loop that ties the dual beam difference frequency to a desired offset. Currently existing opto-electronical phase lock loops (OEPLL) based on electronic components (mixers, amplifiers, etc.), can be used for locking only modest microwave offset frequencies up to about $20 \mathrm{GHz}$ [1]. This practical limit arises due to the unavoidable loop propagation delay in an electronic system (see Fig. 1, left). It would be highly desirable to implement a phase lock loop in which the intermediate frequency (IF) signal, corresponding to the deviation of the optical-beat frequency from the standard frequency of an offset generator, is itself coded onto an optical carrier. This would minimize the loop delay time and at the same time enable a dispersion-free and cross-talk-free delivery of the broadband IF feedback signal to the correcting block of the PLL (see Fig.1, right).

The key to a successful implementation of an all-optical PLL is our recent breakthrough in the conceptualization and experimental demonstration of a laser mixer which manipulates the microwave envelopes of optical carriers. 
OEPLL

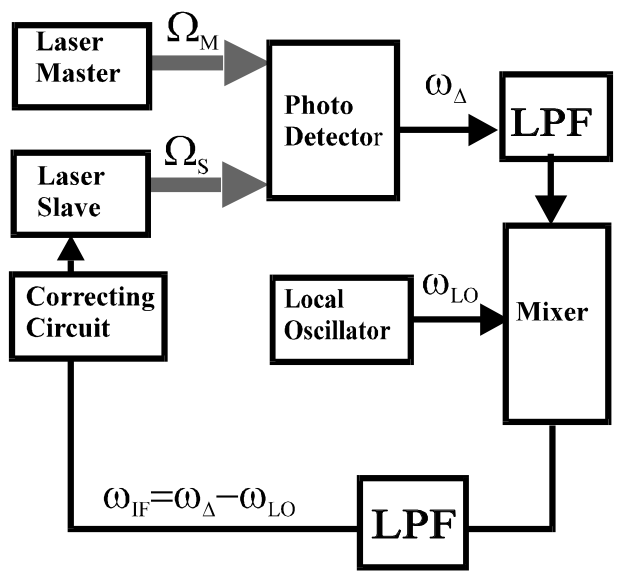

AOPLL

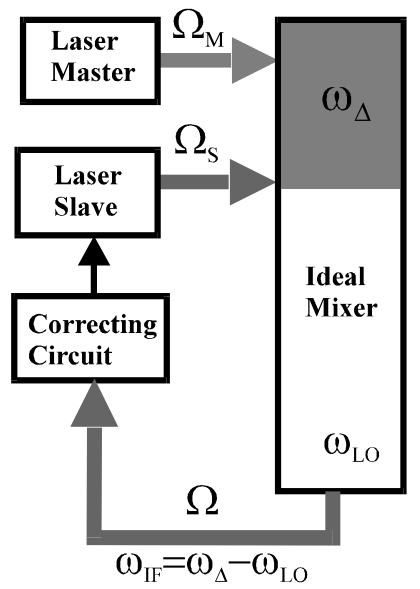

Fig. 1. From opto-electronic to all-optical PLL.

The ideal mixer combines the functions of a photodetector, a local oscillator and a mixer. Black and gray arrows correspond to electrical and optical signals respectively.

\section{Laser Mixer}

Recently, we proposed and experimentally demonstrated [2] a novel type of optoelectronic mixer, the laser-mixer. In this device the microwave signal is encoded in the form of an intensity modulation of the laser optical output. The input signals to be mixed can themselves be either lightwaves modulated in amplitude at $\mathrm{mm}$ wave frequencies or conventional microwaves (or both). The essential physics underlying the concept of the laser-mixer is external modulation of "material" parameters of the laser controlling the propagation of light in the laser cavity, such as the modal gain or optical losses. Consider the rate equation for photon density in the laser cavity:

$$
\frac{d S}{d t}=S\left(\Gamma g-\alpha_{l o s s}\right)
$$

where $\mathrm{g}$ is the material gain, $\Gamma$ is the confinement factor, $\alpha_{\mathrm{loss}}$ is the cavity loss. Modulating two quantities, say, $S$ and $\alpha_{\text {loss }}$

$$
\begin{aligned}
& S=S_{0}+S_{1} \sin \omega_{1} t \\
& \alpha=\alpha_{0}+\alpha_{1} \sin \omega_{2} t
\end{aligned}
$$

we obtain an optical response modulated at the sum and difference frequencies,

$$
\omega=\omega_{1} \pm \omega_{2}
$$


Functionally, this physics is entirely analogous to that in conventional electronic mixers, where mixing is accomplished due to the variation of material parameters, controlling the electric output of the device (capacitance, resistance, etc.). The idea of a parametric control of laser output, combined with a pumping current variation, is relatively new $[3,4]$. Experimentally, an efficient parametric control was demonstrated in a threeterminal quantum-well laser structure, realized in two material systems: GaAs/AlGaAs at the Ioffe Institute and InGaAs/InGaP/GaAs at Bell Laboratories[5,6].

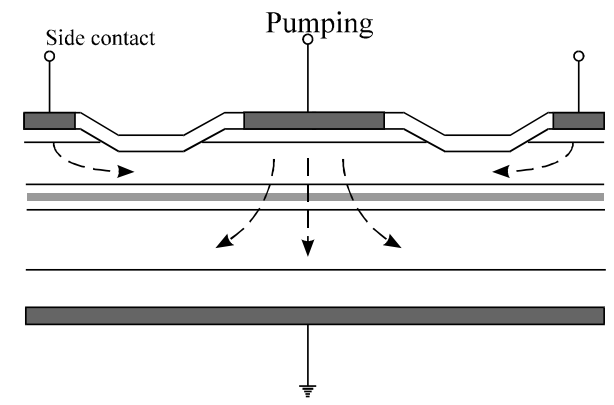

Fig. 2. Three-terminal laser structure with dual modal gain and pumping current control.

This three-terminal structure (Fig. 2) lends itself to a natural use as a laser-mixer with electric inputs. The electric impedance of the parametric input circuit, controlling the modal gain of this laser structure is $50 \mathrm{ohm}$, which is very attractive for microwave applications. Another experimentally demonstrated parametric laser-mixer [2] was based on the variation of cavity loss in a laser with saturable absorber (Fig. 3).

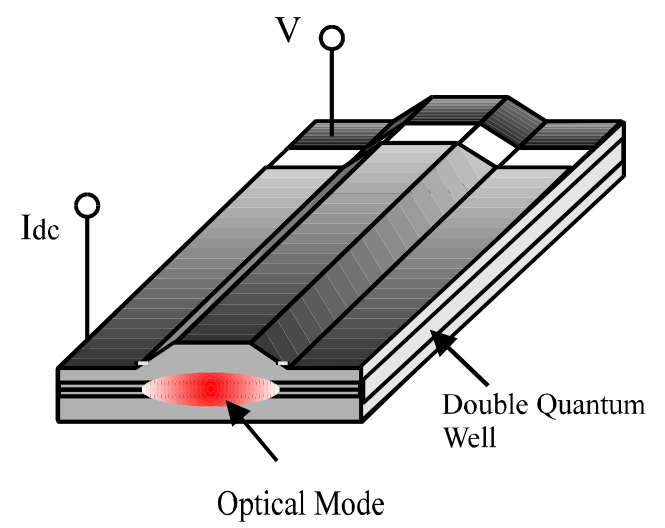

Fig. 3. Laser with saturable absorber in selfpulsating regime is capable of mixing the envelope $w_{D}$ of two optical frequencies $\left(\omega_{\Delta}\right.$ $=\Omega_{1} \Omega_{2}$, ) with its self-pulsation at frequency $\omega_{\mathrm{L}_{\mathrm{O}}} \quad$ Resulting laser output at optical frequency $\Omega$ is modulated at difference frequency $\omega_{\mathrm{I}}=\omega_{\Delta}-\omega_{\mathrm{L}_{\mathrm{O}}}$.

Because of the short (picosecond) recovery time available in saturable absorbers, this type of laser-mixer can take as input optical signals modulated in intensity at frequencies well over $100 \mathrm{GHz}$. Moreover, the optical nature of the input signal closes the entire loop in the optical medium and permits the implementation of all-optical circuits, operating entirely with microwave signals coded as the envelope of an optical carrier.

It should be clearly understood that parametric mixing in semiconductor laser is different in principle from the heterodyne coherent mixing of optical waves. The latter technique is based on the square-law dependence of the optical transition rate on the lightwave 
amplitude | A |. In contrast, with respect to the lightwave intensity| $\left.\mathbf{A}\right|^{2}$ the semiconductor laser is a highly linear element. Mixing of different channels in an optical communication system - which manifests itself as an unwelcome intermodulation distortion - is an exceedingly minor effect (typically less than $-70 \mathrm{dBc}$ ) which arises mostly due to higher-order parametric phenomena [7].

\section{Operation principle of the AOPPL}

Let us return to the AOPLL structure shown in Fig. 1 and discuss its operation principle. Part of the radiation from both the master and the slave lasers is focused on the fast saturable absorber, modulating its transparency at the difference frequency

$$
\omega_{\Delta}=\Omega_{\mathrm{M}}-\Omega_{\mathrm{S}}
$$

and phase $\phi_{\Delta}$. This frequency is mixed with that of mode-locked pulsation in the laser cavity at the the latter frequency $\omega_{\mathrm{LO}}$ can be as high as hundreds of $\mathrm{GHz}[2,3]$. It may be further stabilized by an electronic generator. The intermediate frequency

$$
\omega_{\mathrm{IF}}=\omega_{\Delta}-\omega_{\mathrm{LO}}
$$

is then transmitted to the correcting circuit as an envelope of the optical output of the laser mixer. The inherited phase of the intermediate signal equals $\phi_{\mathrm{IF}}=\phi_{\Delta}-\phi_{\mathrm{LO}}$. This signal is received by a fast photodiode integrated in the correcting circuit, which tunes the slave laser so as to keep $\phi_{\mathrm{IF}}$ constant.

As shown in Fig.1, the electric part of the PLL the correcting circuit is localized in the vicinity of the slave laser. The entire optical circuit can be integrated on a single silicon substrate. At the same time the AOPLL architecture permits a relatively remote positioning of the master and slave lasers, which is advantageous for their stability. We would like to emphasize the universality of the all-optical PLL architecture, rooted in the fundamentally dispersionless transmission of the microwave envelope via optical waveguides. Not only this implies the broad loop bandwidth but it also means that changing the operating microwave frequency does not entail any major revision of the passive optical circuit.

\section{Conclusion}

The laser-mixer whose optical output is modulated in amplitude at the intermediate frequency between two microwave or millimeter wave signals (themselves either optical or electrical) is a new element with a considerable potential for the implementation of optically interconnected systems operating in a very broad range of microwave frequencies. These systems may have a cascade structure and produce simultaneously microwave signals at multiple frequencies. As an example of the new possibilities, consider a system (Fig. 4.) generating a grid of equidistant optical frequencies spaced apart by a given offset frequency. In this system the slave laser of the $n$-th AOPLL cascade plays the role of master for the following $(n+1)$-st cascade. 


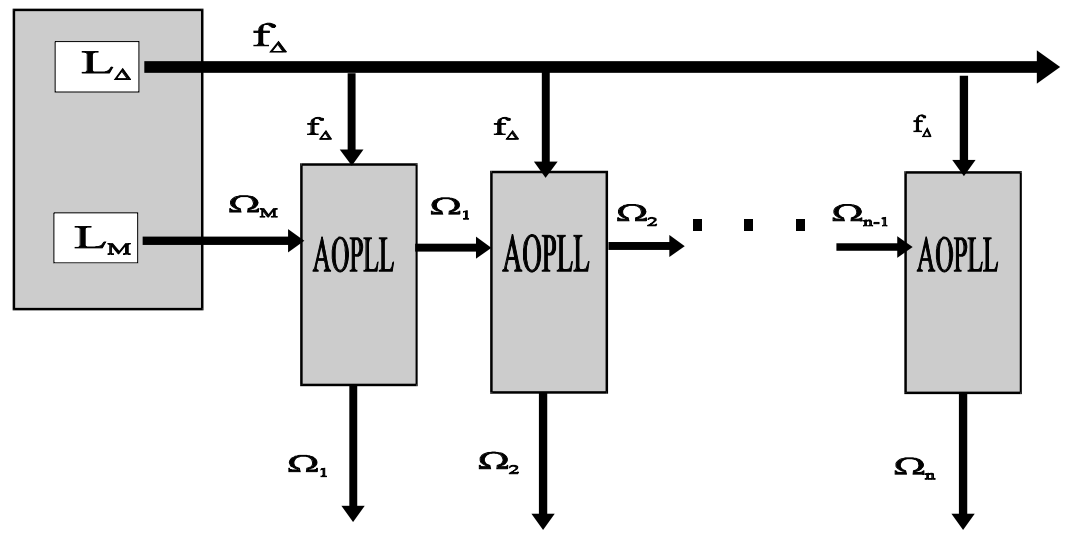

Fig. 4. Block diagram of the source of multiple optical frequencies

A particular example of a system could be an array of optically coherent lasers operating at the same wavelength. In this case, an optical signal emitted by a single laser-master would be split between a number of AOPLL's which control the laser-slave array, thus keeping a given unique offset frequency between the laser-master and each of the laser-slaves.

\section{References}

1. Seeds, A.J. (1993) "Optical Technologies for Phased Array Antennas", IEICE Trans. Electron.E76-C, 198-206.

2. Portnoi E.L., Gorfinkel, V.B., Avrutin, E.A., Thayne, I., Barrow, D.A., Marsh, J.H. and Luryi, S (1995) "Optoelectronic Microwave-Range Frequency Mixing in

Semiconductor Lasers", IEEE J. Select. Top. Quant. Electron., 1, 451-460.

3. Gorfinkel, V.B. and Luryi, S. (1994), "Dual modulation of semiconductor lasers", Physics and Simulation of Optoelectronic Devices, ed. by M. Osinski, Proc. SPIE 2146, 204-209.

4. Gorfinkel, V.B. and Luryi, S. (1994), "Article that comprises a semiconductor laser, and method of operating the article", US Pat. 5,311,526.

5. Gorfinkel, V.B., Kompa, G., Novotny, M., Gurevich, S., Shtengel, G.,Chebunina, I. (1993), "High-frequency modulation of a QW diode laser by dual modal gain and pumping current control" 1993-IEDM Tech. Digest, 933-937.

6. Frommer,A., Luryi, S., Nichols, D.T., Lopata, J. and Hobson, W.S. (1995) "Direct modulation and confinement factor modulation of semiconductor lasers", Appl. Phys. Lett., 67 (Sept 18,).

7. Gorfinkel, V.B. and Luryi, S. (1995), "Fundamental limits for linearity of CATV lasers", IEEE J. Lightwave Technol., 252-260. 